\title{
The large-mass limit of cloudy black holes
}

\author{
Shahar Hod \\ The Ruppin Academic Center, Emeq Hefer 40250, Israel \\ and \\ The Hadassah Institute, Jerusalem 91010, Israel
}

(Dated: November 12, 2018)

\begin{abstract}
The interplay between black holes and fundamental fields has attracted much attention over the years from both physicists and mathematicians. In this paper we study analytically a physical system which is composed of massive scalar fields linearly coupled to a rapidly-rotating Kerr black hole. Using simple arguments, we first show that the coupled black-hole-scalar-field system may possess stationary bound-state resonances (stationary scalar 'clouds') in the bounded regime $1<$ $\mu / m \Omega_{\mathrm{H}}<\sqrt{2}$, where $\mu$ and $m$ are respectively the mass and azimuthal harmonic index of the field, and $\Omega_{\mathrm{H}}$ is the angular velocity of the black-hole horizon. We then show explicitly that these two bounds on the dimensionless ratio $\mu / m \Omega_{\mathrm{H}}$ can be saturated in the asymptotic $m \rightarrow \infty$ limit. In particular, we derive a remarkably simple analytical formula for the resonance mass spectrum of the stationary bound-state scalar clouds in the regime $M \mu \gg 1$ of large field masses: $\mu_{n}=\sqrt{2} m \Omega_{\mathrm{H}}\left[1-\frac{\pi(\mathcal{R}+n)}{m|\ln \tau|}\right]$, where $\tau$ is the dimensionless temperature of the rapidly-rotating (nearextremal) black hole, $\mathcal{R}<1$ is a constant, and $n=0,1,2, \ldots$ is the resonance parameter. In addition, it is shown that, contrary to the flat-space intuition, the effective lengths of the scalar field configurations in the curved black-hole spacetime approach a finite asymptotic value in the large mass $M \mu \gg 1$ limit. In particular, we prove that in the large mass limit, the characteristic length scale of the scalar clouds scales linearly with the black-hole temperature.
\end{abstract}




\section{INTRODUCTION}

When we think about classical black holes, we usually imagine spacetime regions which are bounded by one-way membranes that irreversibly absorb all kinds of radiation and matter fields [1]. This remarkable feature of the classical black-hole horizon suggests that static matter configurations which are made of fundamental fields cannot be supported in the spacetime region exterior to the black-hole horizon. This physical expectation, first discussed by Wheeler [2, 3] more than four decades ago, is known as the 'no-hair' conjecture.

The no-hair conjecture [2, 3], which promoted the black hole to the status of a fundamental object in general relativity [4], has played a key role in the historical development of black-hole physics. Early explorations of its regime of validity have ruled out the existence of asymptotically flat static configurations composed of a black hole nonlinearly coupled to fundamental scalar fields [5], spinor fields [6], and massive vector fields [7].

It should be emphasized, however, that the various 'no hair' theorems [5-7] do not rule out the possible existence of non-static composed black-hole-fundamental-fields configurations. In fact, it has recently [8-10] been proved that linearized stationary scalar configurations (bound-state scalar resonances) can be supported in the exterior regions of rotating black holes [11]. In a very interesting work, Herdeiro and Radu [12] have generalized the results of [8] to the nonlinear regime by numerically solving the nonlinear coupled Einstein-scalar equations, thus providing direct numerical evidence for the existence of these non-static composed black-hole-scalar-field configurations.

The existence of non-static composed black-hole-bosonic-field configurations [8, 12, 13], known as 'cloudy black holes' [14], is a direct consequence of the superradiance phenomenon which characterizes the dynamics of integer-spin fields in spinning black-hole spacetimes [15 18]. In particular, the stationary bound-state scalar configurations [8, 12] are known to be in resonance with the rotating black-hole horizon:

$$
\omega_{\text {field }}=\omega_{\mathrm{c}} \equiv m \Omega_{\mathrm{H}} \quad \text { with } \quad m=1,2,3, \ldots
$$

where $\omega_{\text {field }}$ and $m$ are respectively the orbital frequency of the scalar field and its azimuthal harmonic index, and [19]

$$
\Omega_{\mathrm{H}}=\frac{a}{r_{+}^{2}+a^{2}}
$$

is the angular velocity of the black-hole horizon [20] (here $r_{+}$and $a$ are respectively the 
horizon-radius and the angular-momentum per unit mass of the black hole).

As shown in [15 18], integer-spin modes which are in resonance with the rotating blackhole horizon [that is, bosonic modes which satisfy the resonance condition (10)] do not radiate energy into the central rotating black hole. This fact suggests that, despite the inherent irreversibility associated with the classical black-hole horizon, non-static bosonic field configurations which co-rotate with the central spinning black hole [see Eq. (1)] may survive in the exterior black-hole spacetime.

While the superradiance phenomenon [15-18] insures that scalar fields co-rotating with the black hole with $\omega_{\text {field }}=m \Omega_{\mathrm{H}}$ are not swallowed by it, an additional (and distinct) physical mechanism is required in order to prevent the field from radiating its energy to infinity. For massive fundamental fields, the mutual gravitational attraction between the central black hole and the massive field provides the required confinement mechanism [18]. In particular, for a massive scalar field of mass $\mu$, field modes in the low frequency regime [21]

$$
\omega^{2}<\mu^{2}
$$

are prevented from escaping to infinity [that is, field modes in the low frequency regime (2) decay exponentially fast at asymptotically large distances from the central black hole, see Eq. (14) below].

The main goal of the present study is to explore the physical properties of the composed black-hole-scalar-field configurations [8, 12] ('cloudy' black holes [14]) in the regime

$$
M \mu \gg 1
$$

of large field masses [22] (here $M$ is the mass of the central spinning black hole). As we shall show below, these composed cloudy black-hole configurations can be studied analytically in the regime (4).

The paper is organized as follows: In Sec. II we describe the composed Kerr-scalar-field system and formulate the characteristic field equations which govern the dynamics of the scalar fields in the rotating black-hole spacetime. In Sec. III we use simple arguments in order to show that the coupled black-hole-scalar-field system may possess stationary boundstate resonances in the bounded regime $1<\mu / m \Omega_{\mathrm{H}}<\sqrt{2}$. In Sec. IV we shall derive the resonance condition which characterizes the stationary bound-state resonances of the massive scalar fields in the rapidly-rotating kerr black-hole spacetime. In particular, we 
shall carefully determine the regime of validity of this resonance condition. In Sec. V we shall solve the characteristic resonance condition in the regime $M \mu \gg 1$ of large field masses. In particular, we shall obtain remarkably simple analytical formulas which describe the resonance mass spectrum of the stationary bound-state scalar clouds. In Sec. VI we shall explore the effective spatial lengths of the bound-state scalar field configurations. In particular, we shall show that, contrary to the flat-space intuition, the effective lengths of the scalar clouds approach a finite asymptotic value in the large mass $M \mu \gg 1$ limit. We conclude in Sec. VII with a brief summary of the main results.

\section{DESCRIPTION OF THE SYSTEM}

The composed physical system we shall analyze consists of a scalar field $\Psi$ of mass $\mu$ linearly coupled to a rapidly-spinning Kerr black hole of mass $M$ and angular-momentum per unit mass $a$. In the Boyer-Lindquist coordinate system $(t, r, \theta, \phi)$ the black-hole spacetime is described by the line element [23, 24]

$$
d s^{2}=-\frac{\Delta}{\rho^{2}}\left(d t-a \sin ^{2} \theta d \phi\right)^{2}+\frac{\rho^{2}}{\Delta} d r^{2}+\rho^{2} d \theta^{2}+\frac{\sin ^{2} \theta}{\rho^{2}}\left[a d t-\left(r^{2}+a^{2}\right) d \phi\right]^{2}
$$

where $\Delta \equiv r^{2}-2 M r+a^{2}$ and $\rho \equiv r^{2}+a^{2} \cos ^{2} \theta$. The black-hole (event and inner) horizons are determined by the zeroes of $\Delta$ :

$$
r_{ \pm}=M \pm\left(M^{2}-a^{2}\right)^{1 / 2}
$$

The dynamics of the linearized massive scalar field $\Psi$ in the curved black-hole spacetime is governed by the Klein-Gordon (Teukolsky) wave equation [25, 26]

$$
\left(\nabla^{\nu} \nabla_{\nu}-\mu^{2}\right) \Psi=0
$$

Substituting the field decomposition [27]

$$
\Psi=\sum_{l, m} e^{i m \phi} S_{l m}(\theta ; a \omega) R_{l m}(r ; a, \omega) e^{-i \omega t}
$$

into the characteristic Klein-Gordon wave equation (77), one finds [25, 26] that the angular wave function $S_{l m}$ and the radial wave function $R_{l m}$ satisfy two coupled ordinary differential equations [see Eqs. (9) and (12) below] of the confluent Heun type [25, 26, 28 31]. 
The angular wave functions $S_{l m}(\theta ; a \omega)$, which are known as the spheroidal harmonics, are determined by the angular equation [25, 26, 28 -31]

$$
\frac{1}{\sin \theta} \frac{d}{\theta}\left(\sin \theta \frac{d S_{l m}}{d \theta}\right)+\left[K_{l m}+a^{2}\left(\mu^{2}-\omega^{2}\right)-a^{2}\left(\mu^{2}-\omega^{2}\right) \cos ^{2} \theta-\frac{m^{2}}{\sin ^{2} \theta}\right] S_{l m}=0 .
$$

The regularity conditions at the poles (boundaries) $\theta=0$ and $\theta=\pi$ single out a discrete set of angular eigenvalues $\left\{K_{l m}\right\}$ which are labeled by the integers $l$ and $m$ with $l \geq m$ [30]. In the present study we shall analyze the physical properties of the bound-state scalar resonances in the eikonal (large-mass) regime

$$
l=m \gg 1,
$$

in which case the angular eigenvalues of (9) are given by the simple asymptotic relation [32, 33 ]

$$
K_{m m}=m^{2}-a^{2}\left(\mu^{2}-\omega^{2}\right)+O(m)
$$

The radial Klein-Gordon (Teukolsky) equation is given by [25, 26]

$$
\frac{d}{d r}\left(\Delta \frac{d R}{d r}\right)+U R=0
$$

where

$$
U \equiv \Delta^{-1}\left[\left(r^{2}+a^{2}\right) \omega-m a\right]^{2}+2 m a \omega-K_{l m}-\mu^{2}\left(r^{2}+a^{2}\right) .
$$

Note that the angular eigenvalues $\left\{K_{l m}\left(a \sqrt{\mu^{2}-\omega^{2}}\right)\right\}$ couple the radial field equation (12) to the characteristic spheroidal equation (9) [34].

The bound-state scalar configurations are characterized by radial eigenfunctions which decay exponentially fast at asymptotically large distances from the central black hole [18]:

$$
R(r \rightarrow \infty) \sim \frac{1}{r} e^{-\sqrt{\mu^{2}-\omega^{2}} r},
$$

where $\omega^{2}<\mu^{2}$ [see Eq. (3)]. In addition, we shall impose the physically motivated boundary condition of purely ingoing waves (as measured by a comoving observer) at the outer horizon of the black hole [18]:

$$
R\left(r \rightarrow r_{+}\right) \sim e^{-i\left(\omega-\omega_{c}\right) y}
$$

where the critical field frequency $\omega_{\mathrm{c}}$ is determined in (11) and the "tortoise" radial coordinate $y$ is defined by the relation $d y / d r=\left(r^{2}+a^{2}\right) / \Delta[35]$. 
The two boundary conditions (14) and (15), along with the requirement (1), single out a discrete spectrum of eigen field-masses which characterize the stationary bound-state field configurations in the rotating black-hole spacetime. Below we shall determine analytically this discrete resonance spectrum in the regime $M \mu \gg 1$ of large field masses.

\section{UPPER AND LOWER BOUNDS ON THE ALLOWED FIELD MASSES OF THE STATIONARY SCALAR CLOUDS}

In the present section we shall use simple arguments in order to obtain upper and lower bounds on the allowed field masses of the stationary bound-state scalar clouds. To that end, we shall first define the new radial function [36]

$$
\psi \equiv \Delta^{1 / 2} R
$$

in terms of which the radial Teukolsky (Klein-Gordon) equation (12) takes the form

$$
\frac{d^{2} \psi}{d r^{2}}+\left(\omega_{\mathrm{c}}^{2}-V\right) \psi=0
$$

of a Schrödinger-like wave equation, where

$$
\omega_{\mathrm{c}}^{2}-V=\frac{\Delta U+M^{2}-a^{2}}{\Delta^{2}}
$$

The asymptotic behavior of the effective binding potential $V(r)$ is given by

$$
V(r)=\mu^{2}-\frac{4 M \omega_{\mathrm{c}}^{2}-2 M \mu^{2}}{r}+O\left(\frac{1}{r^{2}}\right)
$$

As discussed in the Introduction, the stationary scalar clouds correspond to bound-state massive scalar resonances which are trapped by the effective binding potential in the blackhole exterior region. For the effective potential (18) to have a trapping well, its asymptotic gradient must be positive:

$$
V^{\prime} \rightarrow 0^{+} \quad \text { as } \quad r \rightarrow \infty
$$

Taking cognizance of Eqs. (19), (3), (19), and (20), one finds that the regime of existence of the stationary bound-state scalar clouds is bounded by

$$
\frac{1}{\sqrt{2}} \mu<m \Omega_{\mathrm{H}}<\mu,
$$


or equivalently

$$
1<\frac{\mu}{m \Omega_{\mathrm{H}}}<\sqrt{2}
$$

Note that the inequality (22) is compatible with the large mass regime (4) for large values of the azimuthal harmonic index $m$ [see Eq. (10)] .

Below we shall show explicitly that these two bounds on the dimensionless ratio $\mu / m \Omega_{\mathrm{H}}$ can be approached arbitrarily close in the asymptotic $M \mu \gg 1$ limit of large field masses.

\section{THE RESONANCE CONDITION AND ITS REGIME OF VALIDITY}

In the present section we shall obtain the characteristic resonance condition for the stationary bound-state resonances of the massive scalar fields in the rapidly-rotating (nearextremal) Kerr black-hole spacetime. These stationary scalar resonances (scalar clouds) correspond to the threshold (critical) frequency

$$
\omega=\omega_{\mathrm{c}}
$$

for superradiant scattering of bosonic fields in the rotating black-hole spacetime [see Eq. (11)].

The resonance condition for stationary scalar clouds in the regime $M \mu=O(1)$ [which corresponds to the regime $m=O(1)$ ] was first derived in [8]. Here we shall generalize the analysis of [8] to the regime $M \mu \gg 1$ of large scalar masses [see Eq. (44)]. In addition, we shall carefully determine the regime of validity of the obtained resonance condition [see Eqs. (50) and (56) below].

Before proceeding, it is worth emphasizing that extra care should be taken in determining the exact regime of validity of the resonance condition in the present analysis, which explores the physical properties of the stationary bound-state scalar clouds in the double asymptotic regime

$$
m \gg 1 \quad \text { with } \quad \tau \equiv \frac{r_{+}-r_{-}}{r_{+}} \ll 1 .
$$

In particular, as we shall show below, it is highly important to determine the exact asymptotic behavior of the combination $m \tau$ in the double limit (24).

It is convenient to define the dimensionless variables [25, 26]

$$
x \equiv \frac{r-r_{+}}{r_{+}} \quad ; \quad k \equiv 2 \omega_{\mathrm{c}} r_{+}
$$


in terms of which the radial Teukolsky equation (12) becomes

$$
x(x+\tau) \frac{d^{2} R}{d x^{2}}+(2 x+\tau) \frac{d R}{d x}+U R=0,
$$

where

$$
U=\frac{(1+x / 2)^{2} k^{2} x}{x+\tau}-K+2 m a \omega_{\mathrm{c}}-\mu^{2}\left[r_{+}^{2}(1+x)^{2}+a^{2}\right] .
$$

We shall first study the behavior of the radial function in the near-horizon region

$$
x \ll 1 .
$$

In this region the radial equation is given by (26) with the effective near-horizon potential $U \rightarrow U_{\text {near }} \equiv k^{2} x /(x+\tau)-K+2 m a \omega_{\mathrm{c}}-\mu^{2}\left(r_{+}^{2}+a^{2}\right)$. The physical solution [that is, the one satisfying the ingoing boundary condition (15) at the black-hole horizon] of Eq. (26) is given by [30, 37]

$$
R(x)=\left(\frac{x}{\tau}+1\right)_{2}^{-i k} F_{1}\left(\frac{1}{2}+i \delta-i k, \frac{1}{2}-i \delta-i k ; 1 ;-x / \tau\right),
$$

where ${ }_{2} F_{1}(a, b ; c ; z)$ is the hypergeometric function [30] and

$$
\delta^{2} \equiv-K-\frac{1}{4}+2 m a \omega_{\mathrm{c}}+k^{2}-\mu^{2}\left(r_{+}^{2}+a^{2}\right)
$$

We shall henceforth consider the case of real $\delta$ [38, 39].

Using Eq. 15.3.7 of [30], one can write the radial solution (29) in the form

$$
\begin{aligned}
R(x)= & \left(\frac{x}{\tau}+1\right)^{-i k}\left[\frac{\Gamma(2 i \delta)}{\Gamma(1 / 2+i \delta-i k) \Gamma(1 / 2+i \delta+i k)}\left(\frac{x}{\tau}\right)^{-1 / 2+i \delta+i k}\right. \\
& \left.\times{ }_{2} F_{1}\left(\frac{1}{2}-i \delta-i k, \frac{1}{2}-i \delta-i k ; 1-2 i \delta ;-\tau / x\right)+(\delta \rightarrow-\delta)\right] .
\end{aligned}
$$

The notation $(\delta \rightarrow-\delta)$ in (31) means "replace $\delta$ by $-\delta$ in the preceding term." In the region

$$
\frac{m \tau}{x} \ll 1
$$

one may use the property (see Eq. 15.1.1 of [30] )

$$
{ }_{2} F_{1}(a, b ; c ; z) \rightarrow 1 \text { for } \frac{a b}{c} \cdot z \rightarrow 0
$$

of the hypergeometric function in order to approximate the radial solution (31) by

$$
R(x)=\frac{\Gamma(2 i \delta)}{\Gamma(1 / 2+i \delta-i k) \Gamma(1 / 2+i \delta+i k)}\left(\frac{x}{\tau}\right)^{-1 / 2+i \delta}+(\delta \rightarrow-\delta) .
$$


Taking cognizance of Eqs. (28) and (32), one realizes that the expression (34) for the radial function is valid in the range

$$
m \tau \ll x \ll 1 .
$$

We shall next study the behavior of the radial function in the region

$$
x \gg \tau,
$$

in which case the radial Teukolsky equation (26) is well approximated by

$$
x^{2} \frac{d^{2} R}{d x^{2}}+2 x \frac{d R}{d x}+V_{\text {far }} R=0
$$

with the effective far-region potential $U \rightarrow U_{\text {far }}=(k+k x / 2)^{2}-K+2 m a \omega_{\mathrm{c}}-\mu^{2}\left[r_{+}^{2}(1+x)^{2}+a^{2}\right]$. The solution of the far-region radial equation (37) is given by [30, 37]:

$$
R(x)=N_{1} \times(2 \epsilon)^{\frac{1}{2}+i \delta} x^{-\frac{1}{2}+i \delta} e^{-\epsilon x}{ }_{1} F_{1}\left(\frac{1}{2}+i \delta-\kappa, 1+2 i \delta, 2 \epsilon x\right)+N_{2} \times(\delta \rightarrow-\delta),
$$

where ${ }_{1} F_{1}(a, b, z)$ is the confluent hypergeometric function [30] and $\left\{N_{1}, N_{2}\right\}$ are normalization constants to be determined below. Here we have defined

$$
\epsilon \equiv \sqrt{\mu^{2}-\omega_{\mathrm{c}}^{2}} r_{+}
$$

and

$$
\kappa \equiv \frac{k^{2} / 2-\left(\mu r_{+}\right)^{2}}{\epsilon}
$$

In the region

$$
m x \ll 1,
$$

one may use the property (see Eq. 13.1.2 of [30])

$$
{ }_{1} F_{1}(a, b, z) \rightarrow 1 \text { for } \frac{a}{b} \cdot z \rightarrow 0
$$

of the confluent hypergeometric function in order to approximate the radial solution (38) by

$$
R(x)=N_{1} \times(2 \epsilon)^{\frac{1}{2}+i \delta} x^{-\frac{1}{2}+i \delta}+N_{2} \times(\delta \rightarrow-\delta) .
$$

Taking cognizance of Eqs. (36) and (41), one realizes that the expression (43) for the radial function is valid in the range

$$
\tau \ll x \ll m^{-1}
$$


Taking cognizance of (35) and (44), one realizes that, for rapidly-rotating (near-extremal) black holes with $\tau \ll 1$ [see Eq. (24)], there is an overlap region

$$
m \tau \ll x \ll m^{-1},
$$

in which both expressions for the radial eigenfunction [see Eqs. (34) and (43)] are valid. Matching the two expressions (34) and (43) in the overlap region (45)), one finds

$$
N_{1}(\delta)=\frac{\Gamma(2 i \delta)}{\Gamma\left(\frac{1}{2}+i \delta-i k\right) \Gamma\left(\frac{1}{2}+i \delta+i k\right)} \tau^{\frac{1}{2}-i \delta}(2 \epsilon)^{-\frac{1}{2}-i \delta} \quad \text { and } \quad N_{2}(\delta)=N_{1}(-\delta)
$$

for the normalization constants of the radial function (38).

Using Eq. 13.5.1 of [30], one finds that the asymptotic $(x \rightarrow \infty)$ behavior of the radial eigenfunction (38) is given by [30]

$$
\begin{aligned}
R(x \rightarrow \infty) \rightarrow & {\left[N_{1} \times(2 \epsilon)^{\kappa} \frac{\Gamma(1+2 i \delta)}{\Gamma\left(\frac{1}{2}+i \delta+\kappa\right)} x^{-1+\kappa}(-1)^{-\frac{1}{2}-i \delta+\kappa}+N_{2} \times(\delta \rightarrow-\delta)\right] e^{-\epsilon x} } \\
& +\left[N_{1} \times(2 \epsilon)^{-\kappa} \frac{\Gamma(1+2 i \delta)}{\Gamma\left(\frac{1}{2}+i \delta-\kappa\right)} x^{-1-\kappa}+N_{2} \times(\delta \rightarrow-\delta)\right] e^{\epsilon x} .
\end{aligned}
$$

The bound-state resonances of the massive scalar fields in the black-hole spacetime are characterized by exponentially decaying (bounded) eigenfunctions at spatial infinity [see Eqs. (3) and (14)]. Thus, the coefficient of the asymptotically exploding exponent $e^{\epsilon x}$ in (47) must vanish:

$$
N_{1} \times(2 \epsilon)^{-\kappa} \frac{\Gamma(1+2 i \delta)}{\Gamma\left(\frac{1}{2}+i \delta-\kappa\right)} x^{-1-\kappa}+N_{2} \times(\delta \rightarrow-\delta)=0 .
$$

Substituting $N_{1}$ and $N_{2}$ from (46) into (48), one obtains the characteristic resonance condition

$$
\left[\frac{\Gamma(-2 i \delta)}{\Gamma(2 i \delta)}\right]^{2} \frac{\Gamma\left(\frac{1}{2}+i \delta-i k\right) \Gamma\left(\frac{1}{2}+i \delta+i k\right) \Gamma\left(\frac{1}{2}+i \delta-\kappa\right)}{\Gamma\left(\frac{1}{2}-i \delta-i k\right) \Gamma\left(\frac{1}{2}-i \delta+i k\right) \Gamma\left(\frac{1}{2}-i \delta-\kappa\right)}(2 \epsilon \tau)^{2 i \delta}=1
$$

for the bound-state resonances of the massive scalar fields in the rapidly-rotating Kerr blackhole spacetime.

It is worth emphasizing again that, for large field masses $(M \mu \gg 1$, or equivalently $m \gg 1$ ), the resonance condition (49) is valid in the asymptotic regime [see Eq. (45)]

$$
\tau \ll m^{-2}
$$

of near-extremal black holes. 
In the regime $M \mu \gg 1$ of large field masses (or equivalently, in the regime $m \gg 1$ ) one can use the following analytical approximations for the various Gamma functions that appear in the resonance condition (49) [30]:

$$
\begin{gathered}
{\left[\frac{\Gamma(-2 i \delta)}{\Gamma(2 i \delta)}\right]^{2}=-\left(\frac{e}{2 \delta}\right)^{8 i \delta}\left[1+O\left(m^{-1}\right)\right],} \\
\frac{\Gamma\left(\frac{1}{2}+i \delta-i k\right)}{\Gamma\left(\frac{1}{2}-i \delta-i k\right)}=e^{\pi \delta} e^{-2 i \delta}(k+\delta)^{i(k+\delta)}(k-\delta)^{-i(k-\delta)}\left[1+O\left(m^{-1}\right)\right], \\
\frac{\Gamma\left(\frac{1}{2}+i \delta+i k\right)}{\Gamma\left(\frac{1}{2}-i \delta+i k\right)}=e^{-\pi \delta} e^{-2 i \delta}(k+\delta)^{i(k+\delta)}(k-\delta)^{-i(k-\delta)}\left[1+O\left(m^{-1}\right)\right],
\end{gathered}
$$

and

$$
\frac{\Gamma\left(\frac{1}{2}+i \delta-\kappa\right)}{\Gamma\left(\frac{1}{2}-i \delta-\kappa\right)}=e^{-2 i \delta}\left(\kappa^{2}+\delta^{2}\right)^{i \delta} e^{2 i \kappa(\theta-\pi)}\left[1+O\left(m^{-1}\right)\right], \quad \text { where } \quad \theta \equiv \arctan (\delta / \kappa) .
$$

Substituting Eqs. (51)-(54) into (49), one obtains the resonance condition

$$
-(k+\delta)^{2 i(k+\delta)}(k-\delta)^{-2 i(k-\delta)}\left(\kappa^{2}+\delta^{2}\right)^{i \delta} e^{2 i \kappa(\theta-\pi)}\left(\frac{e \epsilon \tau}{8 \delta^{4}}\right)^{2 i \delta}=1,
$$

which is valid in the asymptotic regime $M \mu \gg 1$ (or equivalently, in the asymptotic regime $m \gg 1)$. From Eq. (55) one finds [40]

$$
\tau \frac{e \epsilon}{8 \delta}\left(\alpha^{2}-1\right)\left(\frac{\alpha+1}{\alpha-1}\right)^{\alpha}\left(\gamma^{2}+1\right)^{\frac{1}{2}} e^{\gamma(\theta-\pi)} e^{\pi\left(n+\frac{1}{2}\right) / \delta}=1,
$$

where

$$
\alpha \equiv \frac{k}{\delta} \quad ; \quad \gamma \equiv \frac{\kappa}{\delta}
$$

and the resonance parameter $n$ is an integer.

In the next section we shall explicitly show that, the (rather cumbersome) resonance equation (56) can be solved analytically in the two physically interesting regimes: (1) $\mu \lesssim$ $\sqrt{2} \cdot m \Omega_{\mathrm{H}}$, and (2) $\mu \gtrsim m \Omega_{\mathrm{H}}$. [Note that these two mass limits correspond to the two boundaries of the regime of existence (22) of the stationary bound-state scalar clouds].

\section{THE STATIONARY BOUND-STATE RESONANCES OF THE COMPOSED KERR-SCALAR-FIELD SYSTEM}

In the present section we shall derive (remarkably simple) analytical formulas for the dis-

crete mass spectrum, $\{\mu(m ; n)\}$, which characterizes the bound-state scalar resonances (the 
stationary scalar clouds) in the rapidly-rotating Kerr black-hole spacetime. In particular, we shall show below that, in the large-mass regime $M \mu \gg 1$, the bound-state resonances can almost saturate the previously derived (upper and lower) bounds (22) on the allowed field masses.

\section{A. The upper resonance regime of the mass spectrum}

We shall first consider bound-state scalar resonances in the vicinity of the upper bound $\mu<\sqrt{2} m \Omega_{\mathrm{H}}$ [see Eq. (22) $]$. In particular, we shall write $\mu=\sqrt{2} m \Omega_{\mathrm{H}}(1-\Delta / 2)$ with $\Delta \ll 1$, which implies [41]

$$
M \mu=\frac{m}{\sqrt{2}}(1-\Delta / 2)[1+O(\tau)] \quad ; \quad 0 \leq \Delta \ll 1
$$

We shall henceforth assume that $\Delta \gg \tau$ [see Eq. (65) below]. Taking cognizance of Eqs. (11), (25), (30), (39), (40), (54), (57), and (58), one finds [42]

$$
\epsilon=\frac{1}{2} m(1-\Delta) ; \delta=\frac{1}{2} m(1+\Delta) ; \quad \alpha=2(1-\Delta) ; \gamma=2 \Delta ; \theta=\frac{\pi}{2}-2 \Delta .
$$

Substituting (59) into the resonance condition (56), one obtains after some tedious (but straightforward) algebra [43]

$$
\tau \frac{27 e}{8}\left\{1-\Delta\left[2(1+\ln 3)+\pi\left(1+\frac{2 n+1}{m}\right)\right]\right\} e^{\pi(2 n+1) / m}=1 .
$$

Taking the logarithm of both sides of (60) $)$, one finds [43]

$$
\Delta_{n}=\frac{\frac{m}{2 \pi} \ln \left(\frac{27 e}{8} \tau\right)+\frac{1}{2}+n}{\frac{m}{2 \pi}(2+2 \ln 3+\pi)+\frac{1}{2}+n} \quad ; \quad n \geq n_{\min }
$$

where $n_{\min }$ is the smallest integer $n$ for which $\Delta_{n} \geq 0$ [44]] [or equivalently, the smallest integer $n$ for which $m \ln (27 e \tau / 8)+\pi(1+2 n) \geq 0]$.

Denoting

$$
\mathcal{R} \equiv \frac{m}{2 \pi} \ln \left(\frac{27 e}{8} \tau\right)+\frac{1}{2}-\left\lfloor\frac{m}{2 \pi} \ln \left(\frac{27 e}{8} \tau\right)+\frac{1}{2}\right\rfloor
$$

where $\lfloor x\rfloor$ is the floor function (the largest integer less than or equal to $x$ ), one can express (61) in the form

$$
\Delta_{n}=\frac{\mathcal{R}+n}{\frac{m}{2 \pi}\left[2+2 \ln 3+\pi-\ln \left(\frac{27 e}{8} \tau\right)\right]+\mathcal{R}+n} \quad ; \quad n=0,1,2, \ldots
$$


Note that $\mathcal{R}<1$, which implies [45]

$$
\Delta_{n}<\frac{2 \pi(1+n)}{m|\ln \tau|} \ll 1 \quad \text { for } \quad n \ll m|\ln \tau|,
$$

in accord with our assumption [see Eq. (58)] ].

Note that in the extremal limit $\tau \rightarrow 0(|\ln \tau| \gg 1)$ one can approximate (63) by the remarkably compact expression [46]

$$
\Delta_{n}=\frac{2 \pi(\mathcal{R}+n)}{m|\ln \tau|} \quad ; \quad n=0,1,2, \ldots \quad(n \ll m|\ln \tau|) .
$$

Finally, taking cognizance of the relation (58), one finds the simple analytical expression

$$
M \mu_{n}=\frac{m}{\sqrt{2}}\left[1-\frac{\pi(\mathcal{R}+n)}{m|\ln \tau|}\right] \quad ; \quad n=0,1,2, \ldots \quad(n \ll m|\ln \tau|)
$$

for the discrete family of field masses which characterize the stationary bound-state resonances of the composed black-hole-scalar-field system [47].

\section{B. The lower resonance regime of the mass spectrum}

We shall next consider bound-state scalar resonances in the vicinity of the lower bound $\mu>m \Omega_{\mathrm{H}}$ [see Eq. (22)]. In particular, we shall write $\mu=m \Omega_{\mathrm{H}}(1+\nabla)$ with $\nabla \ll 1$, which implies [41]

$$
M \mu=\frac{m}{2}(1+\nabla)[1+O(\tau)] \quad ; \quad 0 \leq \nabla \ll 1 .
$$

Taking cognizance of Eqs. (11), (25), (30), (39), (40), (154), (157), and (67), one finds [48]

$$
\epsilon=m \sqrt{\nabla / 2} ; \quad \delta=\frac{m}{\sqrt{2}}(1-\nabla / 2) ; \quad \alpha=\sqrt{2} ; \gamma=\frac{1}{2 \sqrt{\nabla}} ; \theta=2 \sqrt{\nabla} .
$$

Substituting (68) into the resonance condition (56), one obtains after some tedious (but straightforward) algebra [49]

$$
\tau \frac{(\sqrt{2}+1)^{2 \sqrt{2}} e^{2}}{16} e^{-\pi / 2 \sqrt{\nabla}} e^{\pi(2 n+1) / \sqrt{2} m}=1
$$

Taking the logarithm of both sides of ([69), one finds that $\nabla_{n}$ can be approximated by the remarkably compact expression

$$
\nabla_{n}=\frac{m^{2}}{2(2 n+1)^{2}} \quad \text { for } \quad n \gg m|\ln \tau|
$$


Note that $\nabla_{n} \ll 1$ for $n \gg m$, in accord with our assumption [see Eq. (67)].

Finally, taking cognizance of the relation (67), one finds the simple analytical expression

$$
M \mu_{n}=\frac{m}{2}\left[1+\frac{1}{2}\left(\frac{m}{2 n+1}\right)^{2}\right][1+O(\tau)] \quad ; \quad n \gg m|\ln \tau| .
$$

for the discrete family of field masses which characterize the stationary bound-state resonances of the composed black-hole-scalar-field system [50].

\section{EFFECTIVE LENGTHS OF THE STATIONARY BOUND-STATE SCALAR CLOUDS}

A 'no short hair' theorem for spherically-symmetric static black holes was proved in [51, 52]. This theorem states that, if a spherically-symmetric static black hole has hair, then this hair (i.e. the external fields) must extend beyond the null circular geodesic (the "photonsphere") of the corresponding black-hole spacetime:

$$
r_{\text {field }} \geq r_{\text {null }} .
$$

In the present section we shall test the validity of this 'no short hair' theorem [51] beyond the regime of spherically symmetric static black holes. In particular, we shall now analyze the effective lengths of the stationary bound-state scalar clouds. A rough estimate for the characteristic heights of the scalar clouds is given by the radial position $r=r_{\text {peak }}$ at which the quantity $4 \pi r^{2}|\Psi|^{2}$ attains its maximum value. In Table \ we display the values of the dimensionless peak radii, $x_{\text {peak }}(m) / \tau$, as directly obtained from the radial eigenfunction (29) in the double asymptotic regime of rapidly-rotating $\tau \ll 1$ black holes and large $M \mu \gg 1$ field masses [see Eq. (50)]. The data presented in Table \refer to bound-state scalar clouds which belong to the upper resonance regime (666) of the mass spectrum [53].

From Table I one learns that the effective lengths of the bound-state scalar clouds are a decreasing function of the azimuthal harmonic index $m$ [or equivalently, a decreasing function of the scalar field mass $\mu$, see Eq. (66) $]$. In fact, one finds that the data presented in Table 【is described extremely well by the simple asymptotic formula:

$$
\frac{x_{\text {peak }}(\tau \rightarrow 0, l=m \gg 1)}{\tau} \simeq \alpha+\frac{\beta}{m}+O\left(m^{-2}\right) \quad \text { with } \quad \alpha \simeq 3.14 ; \beta \simeq 14.1 .
$$

What we find most notable is the fact that the leading-order coefficient $\alpha$ in (73) has a finite asymptotic value. This fact suggests that the stationary bound-state scalar clouds are 


\begin{tabular}{|c|c|c|c|c|c|c|}
\hline$l=m$ & 10 & 25 & 50 & 75 & 100 & 125 \\
\hline$x_{\text {peak }}(m) / \tau$ & 4.262 & 3.699 & 3.439 & 3.335 & 3.276 & 3.237 \\
\hline
\end{tabular}

TABLE I: Stationary bound-state resonances of the composed black-hole-scalar-field system in the double asymptotic regime $\tau \ll 1$ with $l=m \gg 1$ [see Eq. (50)]. We display the dimensionless radius, $x_{\text {peak }}(m) / \tau$, corresponding to the radial position $r=r_{\text {peak }}$ at which the quantity $4 \pi r^{2}|\Psi|^{2}$ attains its maximum value. One finds that the effective heights of the scalar clouds, $x_{\text {peak }}(m) / \tau$, decrease monotonically to an asymptotic finite value [see Eq. (174)] in the $m \gg 1(M \mu \gg 1)$ limit.

characterized by the finite asymptotic $(M \mu \rightarrow \infty)$ limit

$$
\frac{x_{\text {peak }}^{\infty}}{\tau} \simeq 3.14
$$

of the dimensionless effective lengths, where $x_{\text {peak }}^{\infty} \equiv x_{\text {peak }}(\tau \rightarrow 0, l=m \gg 1)$. At this point it is worth noting that the equatorial $(l=m \gg 1)$ null circular geodesics of near-extremal $(\tau \rightarrow 0)$ Kerr black holes are characterized by the dimensionless ratio [54]

$$
\frac{x_{\mathrm{null}}}{\tau}=\frac{2-\sqrt{3}}{2 \sqrt{3}} \simeq 0.077
$$

Taking cognizance of Eqs. (74) and (75), one finds

$$
x_{\text {peak }}(m) \geq x_{\text {peak }}^{\infty}>x_{\text {null }}
$$

in the entire range of allowed field masses [55].

We find it remarkable that the composed black-hole-scalar-field configurations conform to the lower bound (72) despite the fact that they do not satisfy the main assumption (namely, spherical symmetry) of the 'no short hair' theorem presented in [51]. This fact may suggest that the lower bound (72) may be of general validity (that is, even beyond the regime of spherically symmetric static black holes).

\section{SUMMARY AND DISCUSSION}

In summary, the physical properties of rapidly-rotating Kerr black holes coupled to stationary bound-state scalar configurations (linearized scalar 'clouds') were studied analytically. To that end, we have solved analytically the Kerr-Klein-Gordon (Teukolsky) wave equation for linearized massive scalar fields in the regime $M \mu \gg 1$ of large field masses. 
The main results obtained in this paper and their physical implications are:

(1) It has been proved that, the regime of existence of the composed black-hole-scalar-field configurations is bounded by $1<\mu / m \Omega_{\mathrm{H}}<\sqrt{2}$ [see Eq. (22)] .

(2) We have shown explicitly that the two (upper and lower) bounds on the dimensionless ratio $\mu / m \Omega_{\mathrm{H}}$ can be saturated in the asymptotic (large-mass) limit $m \gg 1$. In particular, we have derived remarkably simple analytical formulas [see Eqs. (66) and (71)] which describe the discrete resonance spectrum of the stationary bound-state scalar clouds in the regime $M \mu \gg 1$ of large field masses.

(3) It is worth emphasizing that stationary bound-state scalar clouds with $m=O(1)$ [or equivalently, with $M \mu=O(1)]$ are weakly bound to the central black hole in the sense that they are characterized by the relation $M^{2}\left(\mu^{2}-\omega^{2}\right) \ll 1$ [8, 12]. Here, on the other hand, we have shown that stationary scalar clouds in the regime $m \gg 1$ are strongly bound to the central black hole: in particular, it was shown that these bound-state scalar clouds are characterized by the relation $M^{2}\left(\mu^{2}-\omega^{2}\right) \gg 1$.

(4) It has been shown that, contrary to the flat-space intuition, the effective lengths of the stationary bound-state scalar clouds approach a finite asymptotic value (which scales linearly with the black-hole temperature) in the large mass $M \mu \gg 1$ limit [see Eq. (74)]. In particular, we have shown that the non-trivial spatial behavior of these scalar configurations must extend beyond the equatorial null circular geodesic of the corresponding Kerr blackhole spacetime [see Eqs. (74) and (75)] [56, 57].

We find this property of the (non-static, non-spherically symmetric) scalar configurations quite remarkable since the formal proof of the 'no short hair' theorem (172) provided in [51] is restricted to the static sector of spherically-symmetric black holes. To the best of our knowledge, the fact that the composed black-hole-scalar-field configurations conform to the lower bound (72) provides the first direct evidence for a possible general validity [58] of the 'no short hair' property (72) for composed black-hole-fundamental-fields configurations.

\section{ACKNOWLEDGMENTS}

This research is supported by the Carmel Science Foundation. I thank C. A. R. Herdeiro and E. Radu for helpful correspondence. I would also like to thank Yael Oren, Arbel M. 
Ongo and Ayelet B. Lata for stimulating discussions.

[1] We refer here to fundamental fields which are not associated with globally conserved charges.

[2] R. Ruffini and J. A. Wheeler, Phys. Today 24, 30 (1971).

[3] B. Carter, in Black Holes, Proceedings of 1972 Session of Ecole d'ete de Physique Theorique, edited by C. De Witt and B. S. De Witt (Gordon and Breach, New York, 1973).

[4] By 'fundamental object' we mean an object which is characterized by only few conserved parameters, much like the hydrogen atom in quantum mechanics.

[5] J. E. Chase, Commun. Math. Phys. 19, 276 (1970); J. D. Bekenstein, Phys. Rev. Lett. 28, 452 (1972); C. Teitelboim, Lett. Nuovo Cimento 3, 326 (1972); A. E. Mayo and J. D. Bekenstein, Phys. Rev. D 54, 5059 (1996); I. Pena and D. Sudarsky, Class. Quant. Grav. 14, 3131 (1997).

[6] J. Hartle, Phys. Rev. D 3, 2938 (1971); C. Teitelboim, Lett. Nuovo Cimento 3, 397 (1972).

[7] J. D. Bekenstein, Phys. Rev. D 5, 1239 (1972); 5, 2403 (1972); M. Heusler, J. Math. Phys. 33, 3497 (1992); D. Sudarsky, Class. Quantum Grav. 12, 579 (1995).

[8] S. Hod, Phys. Rev. D 86, 104026 (2012) arXiv:1211.3202]; S. Hod, The Euro. Phys. Journal C 73, 2378 (2013) arXiv:1311.5298.

[9] S. Hod, Phys. Rev. D 90, 024051 (2014) arXiv:1406.1179].

[10] S. Hod, Phys. Lett. B 739, 196 (2014) arXiv:1411.2609].

[11] Since non-linear effects tend to stabilize an exterior black-hole hair, we conjectured in [8] the existence of non-static black holes that are nonlinearly coupled to exterior scalar fields.

[12] C. A. R. Herdeiro and E. Radu, Phys. Rev. Lett. 112, 221101 (2014); C. A. R. Herdeiro and E. Radu, Phys. Rev. D 89, 124018 (2014); C. A. R. Herdeiro and E. Radu, arXiv:1405.3696.

[13] Non-static composed black-hole-field configurations are expected to exist for all types of integer-spin (bosonic) fields, see also [12]. This expectation is based on the fact that the superradiance phenomenon is a generic feature of the dynamics of all bosonic fields in rotating black-hole spacetimes.

[14] We shall use the term 'cloudy black holes' to emphasize the fact that these composed blackhole-bosonic-field configurations are made of black holes which are linearly coupled to bosonic matter fields. On the other hand, the term 'hairy black holes' refers to black holes which are coupled to nonlinear matter fields. 
[15] Ya. B. Zel'dovich, Pis'ma Zh. Eksp. Teor. Fiz. 14, 270 (1971) [JETP Lett. 14, 180 (1971)]; Zh. Eksp. Teor. Fiz. 62, 2076 (1972) [Sov. Phys. JETP 35, 1085 (1972)]; A. V. Vilenkin, Phys. Lett. B 78, 301 (1978).

[16] W. H. Press and S. A. Teukolsky, Nature 238, 211 (1972); W. H. Press and S. A. Teukolsky, Astrophys. J. 185, 649 (1973).

[17] V. Cardoso, O. J. C. Dias, J. P. S. Lemos and S. Yoshida, Phys. Rev. D 70, 044039 (2004) [Erratum-ibid. D 70, 049903 (2004)]; J. C. Degollado, C. A. R. Herdeiro, and H. F. Rúnarsson, Phys. Rev. D 88, 063003 (2013); J. C. Degollado and C. A. R. Herdeiro, Phys. Rev. D 89, 063005 (2014); S. Hod, Phys. Rev. D 88, 064055 (2013) [arXiv:1310.6101]; R. Li, arXiv:1404.6309; S. Hod, Phys. Rev. D 88, 124007 (2013) arXiv:1405.1045; S. Hod, Phys. Lett. B 736, 398 (2014); R. Li and J. Zhao, The Euro. Phys. Journal C 743051 (2014).

[18] T. Damour, N. Deruelle and R. Ruffini, Lett. Nuovo Cimento 15, 257 (1976); T. M. Zouros and D. M. Eardley, Annals of physics 118, 139 (1979); S. Detweiler, Phys. Rev. D 22, 2323 (1980); H. Furuhashi and Y. Nambu, Prog. Theor. Phys. 112, 983 (2004); V. Cardoso and J. P. S. Lemos, Phys. Lett. B 621, 219 (2005); V. Cardoso and S. Yoshida, JHEP 0507:009 (2005); S. R. Dolan, Phys. Rev. D 76, 084001 (2007); S. Hod and O. Hod, Phys. Rev. D 81, Rapid communication 061502 (2010) arXiv:0910.0734]; H. R. Beyer, J. Math. Phys. 52, 102502 (2011); Y. S. Myung, Phys. Rev. D 84, 024048 (2011); S. Hod, Phys. Lett. B 713, 505 (2012); J. P. Lee, JHEP 1201, 091 (2012); S. Hod, Phys. Lett. B 718, 1489 (2013) arXiv:1304.6474; R. Brito, V. Cardoso, and P. Pani, Phys. Rev. D 88, 023514 (2013); S. R. Dolan, Phys. Rev. D 87, 124026 (2013); H. Witek, V. Cardoso, A. Ishibashi, and U. Sperhake, Phys. Rev. D 87, 043513 (2013); V. Cardoso, Gen. Relativ. and Gravit. 45, 2079 (2013); J. C. Degollado and C. A. R. Herdeiro, Gen. Rel. Grav. 45, 2483 (2013); R. Li, The Euro. Phys. Journal C 73, 2274 (2013); S. J. Zhang, B. Wang, E. Abdalla, arXiv:1306.0932; H. Witek, arXiv:1307.1145; Y. S. Myung, Phys. Rev. D 88, 104017 (2013); R. Li, Phys. Rev. D 88, 127901 (2013); H. Okawa, H. Witek, and V. Cardoso, Phys. Rev. D 89, 104032 (2014); B. Arderucio, arXiv:1404.3421; M. O. P. Sampaio, C. Herdeiro, M. Wang, Phys. Rev. D 90, 064004 (2014); Y. Brihaye, C. Herdeiro, and E. Radu, Phys. Lett. B 739, 1 (2014); C. L. Benone, L. C. B. Crispino, C. Herdeiro, and E. Radu, arXiv:1409.1593; C. Herdeiro, E. Radu, and H. Runarsson, arXiv:1409.2877.

[19] We use natural units in which $G=c=\hbar=1$. 
[20] Here $\omega_{\mathrm{c}} \equiv m \Omega_{\mathrm{H}}$ is the threshold (critical) frequency for superradiant scattering of bosonic fields in the black-hole spacetime.

[21] Note that the field mass parameter $\mu$ stands for $\mu / \hbar$. Hence, it has the dimensions of $(\text { length })^{-1}$.

[22] The regime (4) of large field masses corresponds to scalar fields whose Compton wavelengths are small on the scale set by the black-hole mass.

[23] S. Chandrasekhar, The Mathematical Theory of Black Holes, (Oxford University Press, New York, 1983).

[24] R. P. Kerr, Phys. Rev. Lett. 11, 237 (1963).

[25] S. A. Teukolsky, Phys. Rev. Lett. 29, 1114 (1972); S. A. Teukolsky, Astrophys. J. 185, 635 (1973).

[26] T. Hartman, W. Song, and A. Strominger, JHEP 1003:118 (2010).

[27] Here $\omega$ is the conserved frequency of the wave field, and $(l, m)$ are respectively the spheroidal harmonic index and the azimuthal harmonic index of the field mode [see Eq. (9) below].

[28] A. Ronveaux, Heun's differential equations. (Oxford University Press, Oxford, UK, 1995); C. Flammer, Spheroidal Wave Functions (Stanford University Press, Stanford, 1957).

[29] P. P. Fiziev, e-print arXiv:0902.1277; R. S. Borissov and P. P. Fiziev, e-print arXiv:0903.3617; P. P. Fiziev, Phys. Rev. D 80, 124001 (2009); P. P. Fiziev, Class. Quant. Grav. 27, 135001 (2010).

[30] M. Abramowitz and I. A. Stegun, Handbook of Mathematical Functions (Dover Publications, New York, 1970).

[31] S. Hod, Phys. Rev. Lett. 100, 121101 (2008) arXiv:0805.3873]; S. Hod, Phys. Lett. B 717, 462 (2012) arXiv:1304.0529]; S. Hod, Phys. Rev. D 87, 064017 (2013) arXiv:1304.4683.

[32] H. Yang, D. A. Nichols, F. Zhang, A. Zimmerman, Z. Zhang and Y. Chen, Phys. Rev. D 86, 104006 (2012).

[33] We shall also assume that $a \sqrt{\mu^{2}-\omega^{2}}<m$, see Eq. (66) below.

[34] We shall henceforth omit the harmonic indexes $l$ and $m$ for brevity.

[35] Note that $r \rightarrow r_{+}$corresponds to $y \rightarrow-\infty$.

[36] S. Hod, Phys. Lett. B 708, 320 (2012) arXiv:1205.1872].

[37] P. M. Morse and H. Feshbach, Methods of Theoretical Physics (McGraw-Hill, New York, 1953).

[38] One can choose $\delta>0$ without loss of generality. 
[39] The case of imaginary $\delta$ values, which is technically much easier, was studied in [8].

[40] Here we have used the relation $-1=e^{i \pi(2 n+1)}$, where the resonance parameter $n$ is an integer.

[41] Here we have used the relation $M \Omega_{\mathrm{H}}=\frac{1}{2}+O(\tau)$ for rapidly-rotating (near-extremal) Kerr black holes [see Eq. (2)].

[42] The correction terms in (59) are of order $O\left(\Delta^{2}, \tau\right)$.

[43] Here we have assumed the relation $n \Delta / m \ll 1$, which is valid in the regime $n \ll m|\ln \tau|^{1 / 2}$ [see Eq. (65) below].

[44] It is worth emphasizing that the validity of (59) is restricted to the $\kappa \geq 0$ regime (or equivalently, to the $\Delta \geq 0$ regime).

[45] Here we have used the fact that $2+2 \ln 3+\pi-\ln (27 e / 8)>0$.

[46] Note that our assumption $\Delta \gg \tau$ corresponds to the requirement $m \tau|\ln \tau| \ll 1$ [see Eq. (65)]. Taking cognizance of (50) and (65), one indeed finds $\tau / \Delta \sim m \tau|\ln \tau| \ll \tau^{1 / 2}|\ln \tau| \rightarrow 0$ in the extremal $\tau \rightarrow 0$ limit.

[47] It is worth emphasizing again that the analytical expression (66) is valid in the upper regime $\mu \lesssim \sqrt{2} m \Omega_{\mathrm{H}}$ of the mass spectrum [see Eq. (58)]. This implies $n \ll m|\ln \tau|$ in (66) .

[48] The correction terms in (68) are of order $O\left(\nabla^{2}, \tau\right)$.

[49] Here we have assumed the relation $n \nabla / m \ll 1$, which is valid in the regime $n \gg m$ [see Eq. (70) below].

[50] It is worth emphasizing again that the analytical expression (71) is valid in the lower regime $\mu \gtrsim m \Omega_{\mathrm{H}}$ of the mass spectrum [see Eq. (67)]. This implies $n \gg m$ in (71).

[51] S. Hod, Phys. Rev. D 84, 124030 (2011) arXiv:1112.3286].

[52] See also D. Núñez, H. Quevedo, and D. Sudarsky, Phys. Rev. Lett. 76, 571 (1996) for an earlier 'no short hair' theorem for spherically symmetric static black holes. It is worth emphasizing, however, that in [10] we have demonstrated explicitly that this earlier version of the theorem may actually fail for non-spherically-symmetric (rotating) black holes.

[53] Our main goal in this section is to test the validity of the lower bound (72) on the effective lengths of the external fields. Thus, here we are interested in the shortest possible stationary scalar clouds (for given values of the field harmonic parameter $m$ ). It is worth noting that, for stationary bound-state scalar clouds which belong to the lower regime (71) of the mass spectrum, one finds characteristic lengthscales which are larger than the ones reported in Table I. This implies that, the smaller is the mass of the scalar field, the longer is the effective 
length of the corresponding bound-state scalar cloud. In particular, for a given value of the azimuthal harmonic index $m$, the data presented in Table I provides the shortest possible effective length of the corresponding stationary scalar cloud.

[54] J. M. Bardeen, W. H. Press, and S. A. Teukolsky, The Astrophys. Jour. 178, 347 (1972).

[55] It is worth emphasizing again that the effective lengths of the bound-state scalar clouds are a decreasing function of the dimensionless product $M \mu$ (or equivalently, a decreasing function of the field harmonic parameter $m$ ), see Table $\amalg$.

[56] It may be interesting to investigate the possible existence of scalar clouds in the Kerr naked singularity spacetime [57]. In particular, it would be interesting to explore the influence of the trapped null geodesics in the Kerr naked singularity spacetime [57] on the scalar clouds.

[57] Z. Stuchlk and J. Schee, Class. Quantum Grav. 27, 215017 (2010).

[58] That is, beyond the regime of spherically-symmetric static black holes studied in [51]. 